\title{
Odontoma: Case series \& report of cases complicated by infection and multiple denticles ${ }^{*}$
}

\author{
Aakarsh V. Jhamb ${ }^{1}$, Rameshwar Dolas ${ }^{2}$, Prashant Pandilwar ${ }^{3}$, Parul A. Jhamb ${ }^{4}$, Sujata Mohanty ${ }^{5}$ \\ ${ }^{1}$ Department of Oral \& Maxillofacial Surgery, Santosh Dental \& Medical College, Delhi, India \\ ${ }^{2}$ Department of Oral \& Maxillofacial Surgery, Oral \& Maxillofacial Surgery, Indore Dental College, Indore, India \\ ${ }^{3}$ Department of Oral \& Maxillofacial Surgery, Govt. Dental College, Nagpur, India \\ ${ }^{4}$ Department of Oral \& Maxillofacial Pathology, SGT Dental College, Hospital \& Research Institute, Delhi, India \\ ${ }^{5}$ Department of Oral \& Maxillofacial Surgery, Maulana Azad Dental College, New Delhi, India \\ Email: drparulmadan@yahoo.co.in, draakarsh@yahoo.co.in
}

Received 22 July 2011; revised 31 August 2011; accepted 19 September 2011

\begin{abstract}
Odontoma is a hamartomatous lesion of odontogenic origin involving both epithelial and mesenchymal tissues. Although it is not an uncommon lesion yet in certain conditions it can lead to complications if left untreated. This is a retrospective review of 30 diagnosed odontoma cases from the archives of Department of Oral \& Maxillofacial Surgery, Government Dental College \& Hospital, Nagpur, India. Out of these 30 cases, 17 were diagnosed as complex odontoma and 13 cases as compound odontoma. Two of these unusual cases have been described which emphasize the importance of early \& appropriate treatment to prevent further morbidity.
\end{abstract}

Keywords: Odontoma; Maxilla; Mandible; Odontogenic Tumor; Hamartoma

\section{INTRODUCTION}

The term odontoma was first used by Paul Broca in 1867 who defined it as "tumors formed by the overgrowth of transitory or complete dental tissues". Howard listed odontoma as the fourth category of supernumerary tooth. However this category is not universally accepted. The term "odontoma" was earlier referred to "any tumor of odontogenic origin” [1].

As per the current understanding odontoma is considered to be a hamartomatous malformation rather than true neoplasm [2]. Odontoma is a dysmorphic proliferation of both epithelial and mesenchymal components of dental tissues which is neither capable of continuous growth nor complete differentiation into an organ of odontogenic origin and does not infiltrate into the surrounding tissues. It is supposed to originate from an ex-

\footnotetext{
*Odontoma-An Overview.
}

traneous bud of odontogenic cells from the dental lamina and is often associated with impacted teeth and retained deciduous teeth. Although specific causes are unknown, but possible hypotheses include infections, trauma, genetic factors and syndromic association. Cahn and Blum hypothesised [3] that ameloblastic fibroma differentiates into odontoma whereas Eversole and Colleagues postulated that the individual mixed odontogenic tumors are incapable of further differentiation [4].

According to the WHO classification, a complex odontoma is a malformation in which all the dental tissues are represented, individual tissues being mainly well formed but occurring in a more or less disorderly pattern; where as a compound odontoma is a malformation in which all the dental tissues are represented in a more orderly pattern than in the complex odontoma so that the lesion consists of many tooth like structures [5].

This study was aimed to present the data on a series of 30 diagnosed cases of odontomas along with a comparative analysis with previous statistics and also to present two unusual cases of odontomas encountered during the study period.

\section{MATERIALS \& METHODS}

All the cases diagnosed as odontoma from 1998 to 2003 were retrieved from the archives of the department of Oral \& Maxillofacial surgery in Government Dental College, Nagpur, India. A total 30 cases were enucleated and analyzed with respect to age, gender and location (Chart 1 and Chart 2). Almost all the cases were submitted to the department of Oral \& Maxillofacial Pathology with the clinical impression of odontoma [6]. Out of these 30 cases; 2 unusual cases with features of infected complex odontoma and another case of multiple synchronous odontoma with about 40 denticles in maxillary arch have been described. 


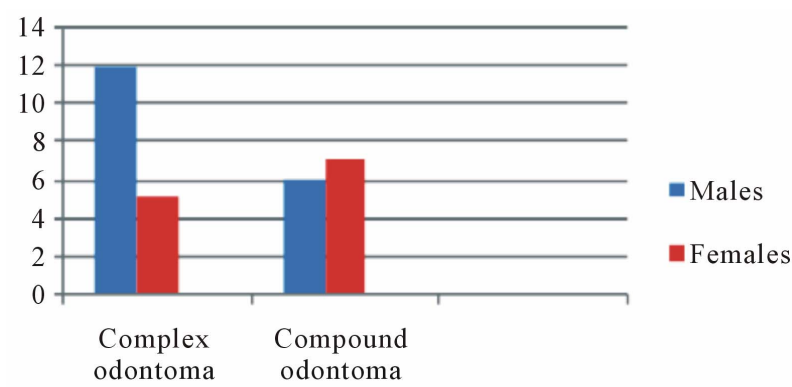

Chart 1. Distribution of the patients according to gender.

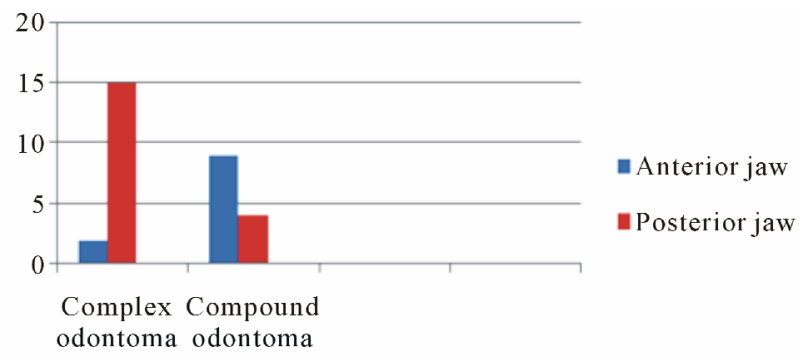

Chart 2. Distribution of the lesions according to site.

\section{RESULTS}

Statistical information relative to the age of the patients, incidence, location and type of odontoma reveals that out of these 30 cases, 17 were complex odontomas and 13 cases were compound odontomas. Mean age of patients with complex odontoma was 29 years, the male: female ratio being $12: 5$ and $88.2 \%$ complex odontoma were seen in posterior mandible. Mean age of patients with compound odontoma was 28.3 years, the male: female ratio being 6:7 and 69.2\% were seen in anterior maxilla. Out of this series; the following two special have been discussed to signify the need for early diagnosis \& treatment of an apparently benign hamartomatous lesion.

\section{CASE REPORTS}

\subsection{Case I}

A 21 year old male patient was referred to the division of Oral \& Maxillofacial surgery with a complaint of pain, swelling and pus discharge from the left cheek. A diffuse intraoral and an associated extra oral bony hard swelling was seen in relation to the left posterior quadrant (Figure 1(a), 1(b)). The swelling was non tender and firm in consistency. The family history and past medical history were not significant. A calcified structure was seen protruding out from the posterior region of left cheek via an infected fistulous tract. Another fistula with a purulent discharge was seen towards the anterior cheek region, around 2 - $3 \mathrm{~cm}$ from the corner of mouth (Figure 1(c)). Intra orally, the swelling involved whole of the lower left quadrant \& extended from retromolar region upto the midline. Overlying gingiva was reddish and swollen. Radiographic examination showed mixed radiolucentradio opaque lesion occupying almost the entire left mandibular posterior quadrant; involving ascending ramus, angle and the body upto first premolar. Cortical

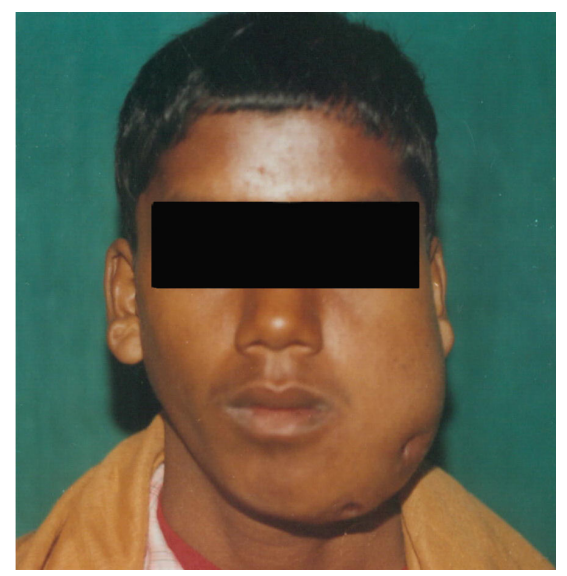

(a)

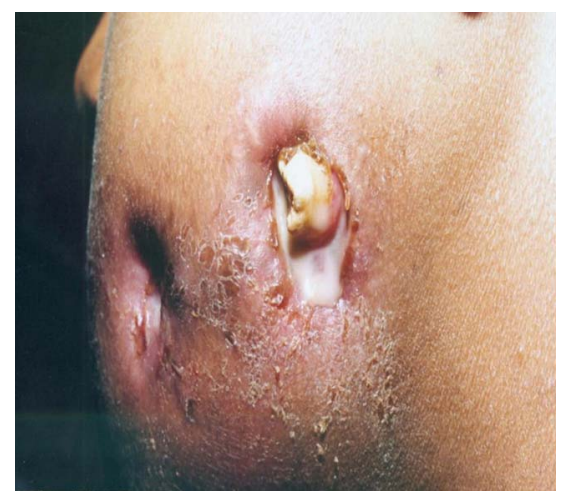

(b)

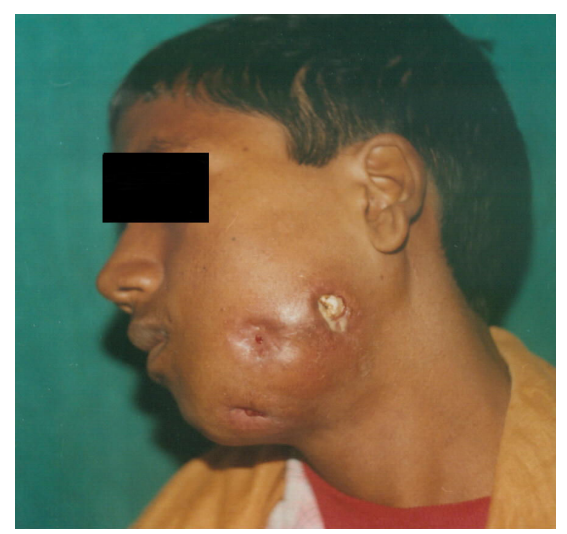

(c)

Figure 1. Clinical photographs of the patient show extra oral swelling on the left cheek with two fistulous tracts having purulent discharge and a tooth like structure protruding out from the cheek near posterior region of mandible. 
bone was expanded and thinned out (Figure 2). A provisional diagnosis of infected odontoma was given and the mass was enucleated under general anesthesia.

The gross specimen revealed hard tissues associated with a soft tissue counterpart \& necrosed areas at the periphery (Figure 3). Histopathological examination revealed haphazardly arranged dentinoid, enamel space \& fibrocellular pulpal component with few vascular channels (Figure 4). At certain foci inflammatory cells infiltrated the fibrovascular tissue (Figure 5).

\subsection{Case II}

A 27 year old male patient was referred to the division of Oral \& Maxillofacial Surgery, GDC, Nagpur; with a complaint of missing teeth, swelling, discomfort, difficulty in chewing and unaesthetic appearance. The patients' family history was non contributory.

Clinical examination revealed that upper and lower incisors were missing; although lower right central and lateral incisors were partly erupted (Figure 6). Panoramic view revealed multiple-tooth like structures of different shapes and sizes in the maxillary anterior region and few globular structures surrounded by radio opaque mass which extended from left canine to the right canine

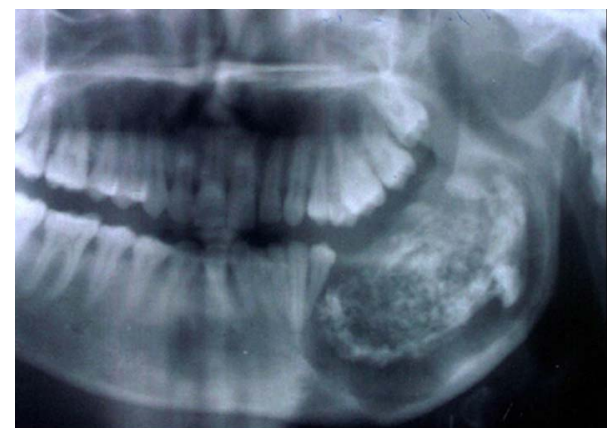

Figure 2. Orthopantamograph of the first case shows a large, ovoid radiolucent lesion on the left side of mandible with a mixed radio opaqueradiolucent mass in the centre.

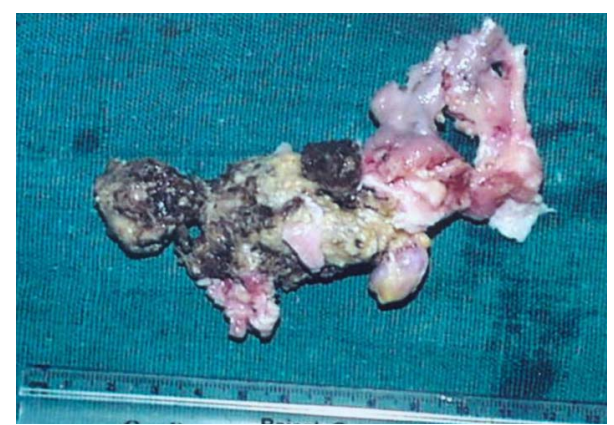

Figure 3. Gross examination of the first case specimen shows creamish brown to pinkish red areas \& pieces of calcified mass as well as soft tissue.

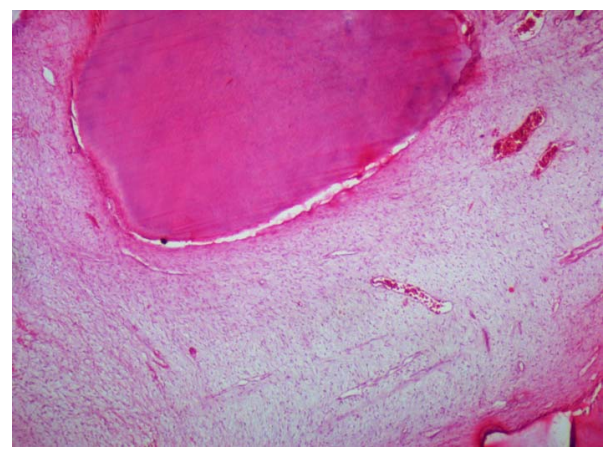

Figure 4. H \& E stained section shows haphazardly arranged dentinoid, enamel space \& pulpal component $(10 \times)$.

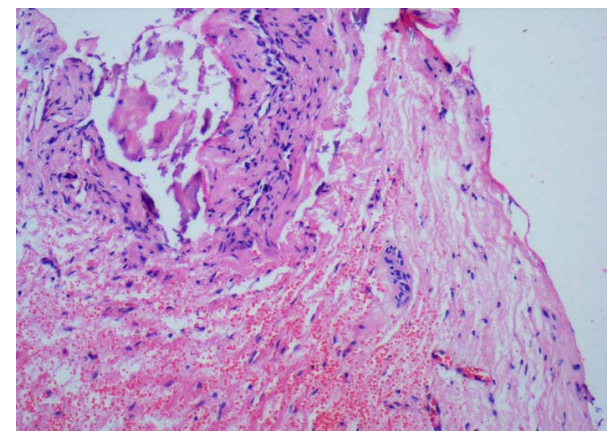

Figure 5. H \& E stained section reveals fibrovascular tissue \& flecks of calcified mass infiltrated by inflammatory cells $(10 \times)$.

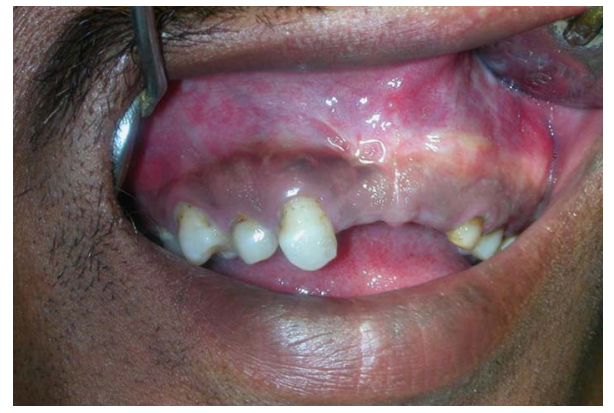

Figure 6. Clinical photograph of the second patient shows missing upper incisors and a small ovoid bony swelling on the alveolar gingiva of the left anterior maxillary region.

in the mandibular anterior region (Figure 7). On the basis of clinical and radiographic findings, a provisional diagnosis of multiple synchronous compound odontoma was made. The routine blood examination revealed no abnormal findings. The serum calcium, phosphorus and alkaline phosphatase levels were also within normal range. Surgical enucleation under local anesthesia was the line of treatment $\&$ a mass of around 40 small compound odontomas in the maxillary anterior lesion extending from the left to the right premolar region was removed. The size of these odontomas varied from $2 \mathrm{~mm}$ 


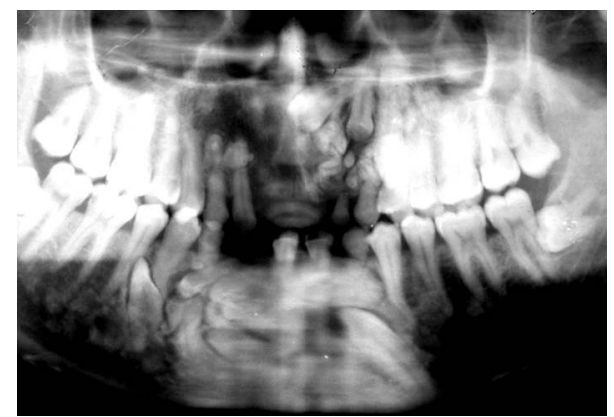

Figure 7. Orthopantamograph of the second case shows mixed radiolucent radio opaque lesions in the maxillary and mandibular anterior region with numerous small-tooth like structures.

to $13 \mathrm{~mm}$. Few of them exhibited fusion and dilacerations (Figure 8). Treatment for the mandibular lesion was rescheduled but patient did not turn up for other investigations and surgery. Microscopic examination showed dentinal matrix with prominent incremental lines \& pulp space (Figure 9). Fibrovascular pulpal tissue could also be appreciated in certain areas (Figure 10).

The mandibular lesion appeared to be larger as compared to the maxillary swelling, thereby suggesting that patient had numerous denticles even in the lower anterior arch. It can be hypothesized that such an aggressive hamartomatous proliferation might have been due to some genetic imbalance or mutation affecting the anterior portion of both the arches simultaneously during intrauterine period.

\section{DISCUSSION}

The aim of selecting and highlighting these two peculiar cases is that they aptly display limits of an entire spectrum which can clinically manifest under this entity. The current opinion states that odontomas are hamartomatous in nature [7]. Three groups have been recognized: ameloblastic fibro odontoma, complex odontoma and compound odontoma. Compound odontomas account for about $2 / 3^{\text {rd }}$ of total number of odontomas and about $10 \%$ of all odontogenic tumors [8]. They are usually symptomless and often prevent the eruption of contiguous teeth. They are frequently discovered incidentally during routine radiographic examination of jaws unlike the mentioned cases. They tend to erupt, especially if they are traumatized or become infected [9]. Most of the odontomas in this series were associated with impacted teeth, retained deciduous teeth, hypodontia, intraoral or extra oral swelling and pain. Out of the presented cases, the case of odontoma associated with erupted calcified mass showed signs of intense infection \& inflammation clinically as well as histopathologically.

According to Neville et al. (1995) compound odon- tomas are twice as commonly observed as the complex odontomas; whereas in our series almost $2 / 3^{\text {rd }}$ cases were that of complex odontomas. Another observation of this series is that compound odontomas were equally distributed among males and females whereas the complex ones had a predilection for males and the latter seems to vary considerably from that observed by Neville et al. [10].

In the present study, it was noted that the distribution

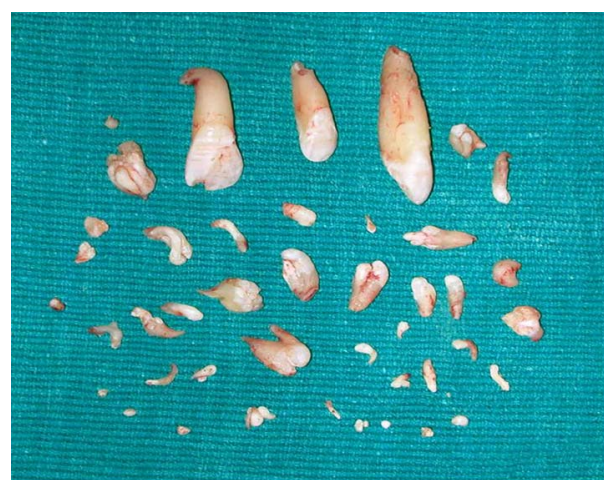

Figure 8. Gross examination of the second case specimen enucleated from the maxilla shows around 40 teeth like structures.

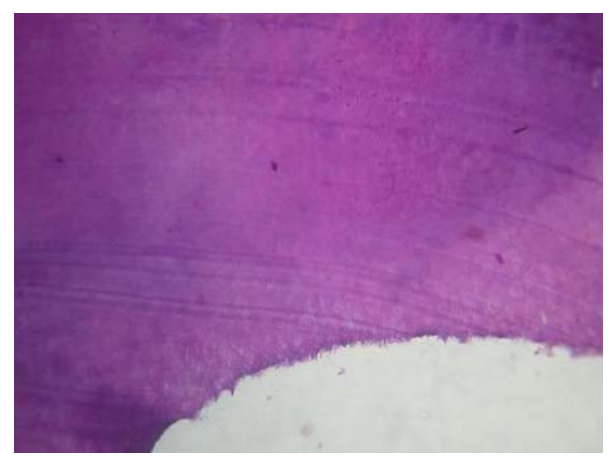

Figure 9. H \& E stained section exhibits dentinal matrix with prominent incremental lines $\&$ pulp space $(10 \times)$.

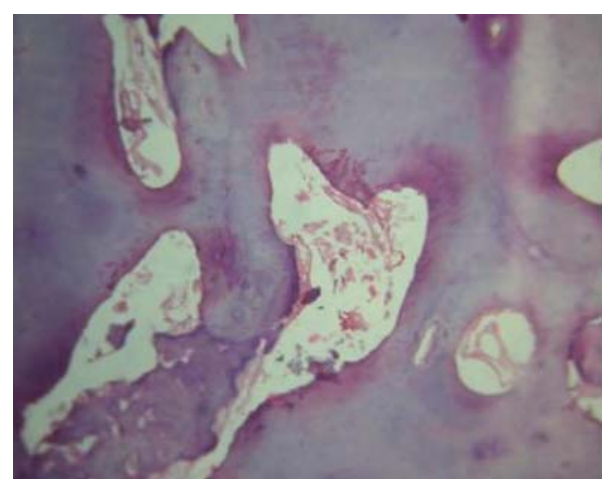

Figure 10. H \& E stained section of fibrovascular pulpal tissue surrounded by dentinal matrix $(10 \times)$. 
of compound and complex odontoma was dependent upon the site of lesion. The compound odontoma occurred predominantly in the anterior jaw; this is in accordance with Slootweg \& Utrechet [4] who stated that the compound odontomas have a predilection for anterior jaw as the odontogenic tissues finish their differentiation earlier in life than in the posterior part of the jaws. Thus, as a corollary majority of the complex odontomas should occur in the posterior regions of either jaw. This corresponds to the findings of Budnick et al. [2]. It was observed that all the cases occurred early in life, mostly in the second and third decade.

Budnick S.D. in 1976 observed that prevalent age for the diagnosis and treatment of odontomas was the second decade of life with a mean age of 14.8 years [2]. Kaugars et al. in 1989 found out that the median age at the time of diagnosis was 16 years. Majority of the cases in their study occurred in the second decade and only a few were diagnosed at or above the age of 30 years [4]. Mean age calculated in the present series for compound and complex odontoma was 28.3 years and 29 years respectively. This can be attributed to the ignorance regarding dental diseases among rural population in the Indian subcontinent and inaccessibility to avail appropriate \& economical treatment. Thus, the developing nations need to implement mass screening \& public awareness programmes as various common lesions are left as such.

In a review of 104 cases of odontomas, Owens et al. identified 67 (64.4\%) compound, 32 (31.0\%) complex, and 5 (4.6\%) diagnoses of both compound and complex odontomas [11]. However, multiple odontomas with extensive involvement of jaws are found very rarely in humans and their prevalence is unknown.

As stated previously, one case in the present analysis was that of infected complex odontoma; a long standing lesion which became aggressive due to secondary infec- tion. The odontoma appeared to protrude out of the left cheek with purulent discharge through a fistulous tract \& an apparent space infection that could have lead to cellulitis.

Another unusual presentation was that of multiple odontomas with more than 40 denticles enucleated from a single lesion in the maxillary anterior region \& a larger synchronous lesion in the mandibular anterior arch that could not be enucleated. The English literature reveals that Sharma et al. (2010) reported a case of compound composite odontoma with 37 denticles.

A review of English literature showed 12 cases of multiple compound odontomas excluding this report; such cases are at times associated with Cleido cranial dysostosis or Gardner's syndrome (GS). Patients affected by GS are at a greater risk of developing cancer in various organs; therefore surveillance of such patients is recommended. Dental anomalies usually seen in GS are impacted teeth, supernumerary teeth, odontomas, and congenitally missing teeth $[12,13]$. According to Philipsen and Reichart the compound odontoma represents a histomorphological malformation which is closely related to the pathological process producing hyperdontia, multiple schizodontia or hyperactivity of dental lamina. The treatment of choice for compound odontomas continues to be conservative surgical removal.

Recurrence is generally not observed if the associated lining epithelium is removed intact (Kaban and Troulis, 1990) [10]. The post operative morbidity is low and the results are predictable after thorough removal of the tumor [11].

These cases signify the severity of naive appearing hamartomatous lesions like odontomas and the complications they can present if left as such. Although the treatment modality is enucleation; but this has to be altered in cases of syndromic association \& occurence of complex odontoma with ameloblastic fibro odontoma. Such long standing patients have greater chances of developing ameloblastic fibrosarcoma. Clinically; a complex odontoma may not be differentiated from a hamartomatous or a neoplastic lesion and a compound odontoma may cause excessive morbidity during secondary infection as elaborated in these reports.

\section{REFERENCES}

[1] Howard, R.D. (1967) The unerupted incisor. A study of the post operative eruptive history of incisors delayed in their eruption by supernumerary teeth. Dental Practitioner and Dental Record, 17, 332-41.

[2] Budnick, S.D. (1976) Compound and complex odontoma. Oral Surgery, Oral Medicine, Oral Pathology, 42, 501-505. doi:10.1016/0030-4220(76)90297-8

[3] Hawkins, P.L. and Sadeghi, E.M. (1986) Ameloblastic fibro- odontoma: Report of case. Journal of Oral and Maxillofacial Surgery, 44, 1014-1019. doi:10.1016/S0278-2391(86)80059-3

[4] Slootweg, P.J. (1981) An analysis of the interrelationship of the mixed odontogenic tumors-Ameloblastic fibroma, ameloblastic fibro-odontoma, and the odontomas. Oral Surgery, Oral Medicine, Oral Pathology, 51, 266-276. doi:10.1016/0030-4220(81)90056-6

[5] Takeda, Y. and Tomich, C.E. (2005) Ameloblastic fibroodontoma. In: World Health Organization Classification of Tumors (WHO). International Agency for Research on Cancer, IARC Press, Lyon, 309.

[6] George, K.E., Michael, M.E. and Louis, A.M. (1989) Odontomas. Oral Surgery, Oral Medicine, Oral Pathology, 67, 172-176. doi:10.1016/0030-4220(89)90324-1

[7] Ajike, S.O. and Adekeye, E.O. (2000) Multiple odon tomas in the facial bones: A case report. International Journal of Oral and Maxillofacial Surgery, 29, 443-444. doi:10.1016/S0901-5027(00)80077-6

[8] Okada, H., Yamamoto, H. and Tilakaratne, W.M. (2007) 
Odontogenic tumors in Sri Lanka: Analysis of 226 cases. Journal of Oral and Maxillofacial Surgery, 65, 875-882. doi:10.1016/j.joms.2006.06.293

[9] Olech, E. (1963) Complex composite odontoma. Oral Surgery, Oral Medicine, Oral Pathology, 16, 960-964.

[10] Sharma, U., Sharma, R., Gulati, A., Yadav, R. and Gauba, K. (2010) Compound composite odontoma with unusual number of denticles-A rare entity. Saudi Dental Journal, 22, 145-149. doi:10.1016/j.sdentj.2010.04.009

[11] Korpi, J.T, Kainulainen, V.T, George, K.B., and Oikarinen, S. (2009) Removal of large complex odontoma using le fort I Osteotomy. Journal of Oral and Maxillofa- cial Surgery, 67, 2018-2021. doi:10.1016/j.joms.2009.04.105

[12] Lee, B.D., Lee, W., Oh, S.H., et al. (2009) A case report of Gardner syndrome with hereditary widespread osteomatous jaw lesions. Oral Surgery, Oral Medicine, Oral Pathology, Oral Radiology and Endodontology, 107, e68-e72. doi:10.1016/j.tripleo.2008.10.018

[13] Iwamoto, O., Harada, H., Kusukawa, J. and Kameyama, T. (1999) Multiple odontomas of the Mandible: A case Report. Journal of Oral and Maxillofacial Surgery, 57, 338-341. 